\title{
STRONG CONVERGENCE OF MARTINGALES IN VON NEUMANN ALGEBRAS
}

\author{
MAKOTO TSUKADA
}

\begin{abstract}
In this paper we prove strong and $L^{1}$-norm convergence of martingales with respect to a faithful normal semifinite weight on a von Neumann algebra.
\end{abstract}

1. Introduction. Let $M$ be a von Neumann algebra and $\varphi$ be a faithful normal semifinite weight on $M_{+}$(the positive cone of $M$ ). We denote by $n_{\varphi}$ the set of all elements $x \in M$ with $\varphi\left(x^{*} x\right)<+\infty$ and by $m_{\varphi}$ the linear span of $n_{\varphi}^{*} n_{\varphi}$. Then $\varphi$ is uniquely extended to a linear functional on $\mathrm{m}_{\varphi}$ and we also denote it by $\varphi$. For a von Neumann subalgebra $N$ of $M$, if there exists a faithful $\sigma$-weakly continuous projection $\varepsilon$ of norm one from $M$ onto $N$ such that $\varphi(x)=\varphi(\varepsilon(x))$ for every $x \in \mathfrak{m}_{\varphi}$, we call $\varepsilon$ the conditional expectation onto $N$ with respect to $\varphi$. Takesaki [6] proved that there exists a unique conditional expectation onto $N$ with respect to $\varphi$ if and only if $\varphi$ is semifinite on $N$ (i.e., $N \cap \mathrm{m}_{\varphi}$ is $\sigma$-weakly dense in $N$ ) and $N$ is globally invariant under the modular automorphism group associated with $\varphi$.

Let $M_{*}$ be the predual of $M$ (i.e., the set of all $\sigma$-weakly continuous linear functionals on $M$ ). Suppose that $N$ is a von Neumann subalgebra of $M$ such that the conditional expectation $\varepsilon$ onto $N$ with respect to $\varphi$ exists. Then we define $L^{1}(N ; \varepsilon)$ as the set of all elements $\psi \in M_{*}$ with $\psi=\psi \circ \varepsilon$. For any $\psi \in M_{*}$ we define $\varepsilon^{*}(\psi)=$ $\psi \circ \varepsilon$.

THEOREM 1. $L^{1}(N ; \varepsilon)$ is isometrically isomorphic to the predual of $N$. Moreover $\varepsilon^{*}$ is a projection of norm one from $M_{*}$ onto $L^{1}(N ; \varepsilon)$.

Proof. Let $N_{*}$ be the predual of $N$. For any $\psi \in N_{*}$ we define $\iota(\psi)=\psi \circ \varepsilon$. Then $\iota(\psi) \in L^{\prime}(N ; \varepsilon)$ and

$$
\|\psi\|=\sup _{\substack{x \in N \\\|x\| \leqslant 1}}|\psi(x)|=\sup _{\substack{x \in M \\\|x\| \leqslant 1}}|\psi(\varepsilon(x))|=\|\iota(\psi)\|,
$$

because $\varepsilon$ is a projection of norm one from $M$ onto $N$. Moreover $\iota\left(\psi^{\prime}{ }_{N}\right)=\psi$ for every $\psi \in L^{1}(N ; \varepsilon)$. Hence $\iota$ is an isometrical isomorphism from $N_{*}$ onto $L^{1}(N ; \varepsilon)$. On the other hand for any $\psi \in M_{*}$ it follows that

$$
\left\|\varepsilon^{*}(\psi)\right\|=\sup _{\substack{x \in M \\\|x\| \leqslant 1}}|\psi(\varepsilon(x))| \leqslant \sup _{\substack{x \in M \\\|x\| \leqslant 1}}|\psi(x)|=\|\psi\| .
$$

Thus $\varepsilon^{*}$ is of norm one. It is clear that $\varepsilon^{*}$ is a projection from $M_{*}$ onto $L^{1}(N ; \varepsilon)$.

Received by the editors March 29, 1982 and, in revised form, September 28, 1982.

1980 Mathematics Subject Classification. Primary 47D25.

Key words and phrases. Martingale, von Neumann algebra, faithful normal semifinite weight, conditional expectation. 
Suppose that $\varphi$ is a state. Goldstein [4] embedded $M$ into $M_{*}$ and defined $L^{1}(N)$ as the closure of $N$ in $M_{*}$ for any von Neumann subalgebra $N$ of $M$. If the conditional expectation $\varepsilon$ onto $N$ with respect to $\varphi$ exists, $L^{1}(N)$ coincides with $L^{1}(N ; \varepsilon)$ defined as above. In that paper he proved the strong convergence of martingales. We generalize this to the case in which $\varphi$ is a weight.

2. Increasing martingales. In this section we fix an increasing net $\left\{N_{\alpha}\right\}$ of von Neumann subalgebras of $M$ such that the conditional expectation $\varepsilon_{\alpha}$ onto $N_{\alpha}$ with respect to $\varphi$ exists for every $\alpha$.

THEOREM 2. The conditional expectation $\varepsilon_{\infty}$ onto $\bigvee_{\alpha} N_{\alpha}$ with respect to $\varphi$ exists and the following assertions are satisfied:

(i) $\left\{\varepsilon_{\alpha}(x)\right\}$ converges to $\varepsilon_{\infty}(x)$ in the $s^{*}$-topology for every $x \in M$;

(ii) $\left\{\varepsilon_{\alpha}^{*}(\psi)\right\}$ converges to $\varepsilon_{\infty}^{*}(\psi)$ in the norm for every $\psi \in M_{*}$.

Proof. Since $\varphi$ is semifinite on $N_{\alpha}$ and $N_{\alpha}$ is globally invariant under the modular automorphism group associated with $\varphi$ for every $\alpha, \varphi$ is semifinite on $\bigvee_{\alpha} N_{\alpha}$ and $\bigvee_{\alpha} N_{\alpha}$ is globally invariant under the modular automorphism group. Hence there exists a unique conditional expectation $\varepsilon_{\infty}$ onto $\bigvee_{\alpha} N_{\alpha}$ with respect to $\varphi$. By the uniqueness of the conditional expectation $\varepsilon_{\alpha}=\varepsilon_{\alpha} \circ \varepsilon_{\infty}$ for every $\alpha$. Therefore to prove (i) and (ii) we may assume that $M=\bigvee_{\alpha} N_{\alpha}$ without loss of generality.

(i) We first prove that if $\varepsilon_{\alpha}(x)=0$ for every $\alpha$, then $x=0$. Assume that $\varepsilon_{\alpha}(x)=0$ for every $\alpha$. We fix an index $\alpha_{0}$ and for any $\psi \in M_{*}$ we put $\hat{\psi}=\psi \circ \varepsilon_{\alpha_{0}}$. Then for any $\alpha \geqslant \alpha_{0}$ and $a \in N_{\alpha}$ we have $\hat{\psi}(a x)=\hat{\psi}\left(\varepsilon_{\alpha}(a x)\right)=\hat{\psi}\left(a \varepsilon_{\alpha}(x)\right)=0$. Therefore $\hat{\psi}(a x)=0$ for any $a \in \cup_{\alpha} N_{\alpha}$. Since $\cup_{\alpha} N_{\alpha}$ is $\sigma$-weakly dense in $M$, we have $\hat{\psi}(a x)=0$ for any $a \in M$. Hence $\psi\left(\varepsilon_{\alpha_{0}}\left(x^{*} x\right)\right)=\hat{\psi}\left(x^{*} x\right)=0$. Since $\psi \in M_{*}$ is arbitrary, $\varepsilon_{\alpha_{0}}\left(x^{*} x\right)=0$, and since $\varepsilon_{\alpha_{0}}$ is faithful, it follows that $x=0$. Next we prove that $\left\{\varepsilon_{\alpha}(x)\right\}$ converges to $x$ in the $\sigma$-weak topology for any $x \in M$. Since $\left\{\varepsilon_{\alpha}(x)\right\}$ is uniformly bounded, for any subnet $\left\{\varepsilon_{\alpha^{\prime}}(x)\right\}$ there exists a subnet $\left\{\varepsilon_{\alpha^{\prime \prime}}(x)\right\}$ which converges to some $y \in M$ in the $\sigma$-weak topology. For every $\alpha$, because of $\sigma$-weak continuity of $\varepsilon_{\alpha}$, it follows that $\varepsilon_{\alpha}\left(\varepsilon_{\alpha^{\prime \prime}}(x)\right)$ tends to $\varepsilon_{\alpha}(y)$ as $\alpha^{\prime \prime} \uparrow$ in the $\sigma$-weak topology. Here for sufficiently large $\alpha^{\prime \prime}$ we have $\varepsilon_{\alpha}\left(\varepsilon_{\alpha^{\prime \prime}}(x)\right)=\varepsilon_{\alpha}(x)$. Hence $\varepsilon_{\alpha}(x)=$ $\varepsilon_{\alpha}(y)$ for every $\alpha$ and we have $x=y$ by the fact that is proved above. Thus $\left\{\varepsilon_{\alpha}(x)\right\}$ converges to $x$ in the $\sigma$-weak topology. Moreover for any $\psi \in M_{*}^{+}$it follows that

$$
\begin{aligned}
0 & \leqslant \psi\left(\left(\varepsilon_{\alpha}(x)-x\right)^{*}\left(\varepsilon_{\alpha}(x)-x\right)\right) \\
& =\psi\left(\varepsilon_{\alpha}(x)^{*} \varepsilon_{\alpha}(x)\right)-\psi\left(\varepsilon_{\alpha}(x)^{*} x\right)-\psi\left(x^{*} \varepsilon_{\alpha}(x)\right)+\psi\left(x^{*} x\right) \\
& \leqslant \psi\left(\varepsilon_{\alpha}\left(x^{*} x\right)\right)-\psi\left(\varepsilon_{\alpha}(x)^{*} x\right)-\psi\left(x^{*} \varepsilon_{\alpha}(x)\right)+\psi\left(x^{*} x\right) \\
& \rightarrow 0 \quad(\text { as } \alpha \uparrow)
\end{aligned}
$$

$\psi\left(\left(\varepsilon_{\alpha}(x)-x\right)\left(\varepsilon_{\alpha}(x)-x\right)^{*}\right)$ tends to 0 as $\alpha \uparrow$ in the same way and we have (i).

(ii) Since $\left\{\varepsilon_{\alpha}(x)\right\}$ converges to $x$ in the $\sigma$-weak topology for any $x \in M,\left\{\varepsilon_{\alpha}^{*}(\psi)\right\}$ converges to $\psi$ in the weak topology for any $\psi \in M_{*}$. Therefore $\cup_{\alpha} L^{1}\left(N_{\alpha} ; \varepsilon_{\alpha}\right)$ is weakly dense in $M_{*}$ and so in the norm. Hence we have (ii) by the standard argument. 
REMARK. The strong convergence of martingales was proved by Connes [2] for the case in which $M$ is $\sigma$-finite, and by Lance [5] for the case in which $\vee_{\alpha} N_{\alpha}$ is semifinite.

3. Decreasing martingales. In this section we fix a decreasing net $\left\{N_{\alpha}\right\}$ of von Neumann subalgebras of $M$ such that the conditional expectation $\varepsilon_{\alpha}$ onto $N_{\alpha}$ with respect to $\varphi$ exists for every $\alpha$.

THEOREM 3. Suppose that $\varphi$ is semifinite on $\bigcap_{\alpha} N_{\alpha}$. Then the conditional expectation $\varepsilon_{\infty}$ onto $\bigcap_{\alpha} N_{\alpha}$ with respect to $\varphi$ exists and the following assertions are satisfied:

(i) $\left\{\varepsilon_{\alpha}(x)\right\}$ converges to $\varepsilon_{\infty}(x)$ in the $s^{*}$-topology for every $x \in M$;

(ii) $\left\{\varepsilon_{\alpha}^{*}(\psi)\right\}$ converges to $\varepsilon_{\infty}^{*}(\psi)$ in the norm for every $\psi \in M_{*}$.

Proof. Since $N_{\alpha}$ is globally invariant under the modular automorphism group associated with $\varphi$ for every $\alpha$, so is $\bigcap_{\alpha} N_{\alpha}$, and since $\varphi$ is semifinite on $\bigcap_{\alpha} N_{\alpha}$, there exists a unique conditional expectation $\varepsilon_{\infty}$ onto $\bigcap_{\alpha} N_{\alpha}$ with respect to $\varphi$. By the uniqueness of the conditional expectation, $\varepsilon_{\infty}=\varepsilon_{\infty} \circ \varepsilon_{\alpha}$ for every $\alpha$.

(ii) Let any $\psi \in M_{*}$ be fixed. Then

$$
\begin{aligned}
\left\|\varepsilon_{\alpha}^{*}(\psi)-\varepsilon_{\infty}^{*}(\psi)\right\| & =\sup _{\substack{x \in M \\
\|x\| \leqslant 1}}\left|\psi\left(\varepsilon_{\alpha}(x)\right)-\psi\left(\varepsilon_{\infty}(x)\right)\right| \\
& =\sup _{\substack{x \in M \\
\|x\| \leqslant 1}}\left|\psi\left(\varepsilon_{\alpha}(x)\right)-\psi\left(\varepsilon_{\infty}\left(\varepsilon_{\alpha}(x)\right)\right)\right| \\
& =\sup _{\substack{x \in N_{\alpha} \\
\|x\| \leqslant 1}}\left|\psi(x)-\psi\left(\varepsilon_{\infty}(x)\right)\right|=\left|\psi\left(x_{\alpha}\right)-\psi\left(\varepsilon_{\infty}\left(x_{\alpha}\right)\right)\right|
\end{aligned}
$$

for some $x_{\alpha} \in N_{\alpha}$ with $\left\|x_{\alpha}\right\| \leqslant 1$. Since $\left\{x_{\alpha}\right\}$ is uniformly bounded, for any subnet $\left\{x_{\alpha^{\prime}}\right\}$ there exists a subnet $\left\{x_{\alpha^{\prime \prime}}\right\}$ which converges to some $x_{\infty} \in M$ in the $\sigma$-weak topology. Then $x_{\infty} \in \bigcap_{\alpha} N_{\alpha}$ and $\left|\psi\left(x_{\alpha^{\prime \prime}}\right)-\psi\left(\varepsilon_{\infty}\left(x_{\alpha^{\prime \prime}}\right)\right)\right|$ tends to 0 as $\alpha^{\prime \prime} \uparrow$. Therefore it follows that $\left\{\varepsilon_{\alpha}^{*}(\psi)\right\}$ converges to $\varepsilon_{\infty}^{*}(\psi)$ in the norm.

(i) It can be easily seen that $\left\{\varepsilon_{\alpha}(x)\right\}$ converges to $\varepsilon_{\infty}(x)$ in the $\sigma$-weak topology in the same way as the proof of Theorem 2(i). For any $\psi \in M_{*}^{+}$we have

$$
\begin{aligned}
\psi\left(\left(\varepsilon_{\alpha}(x)-\varepsilon_{\infty}(x)\right)^{*}\left(\varepsilon_{\alpha}(x)-\varepsilon_{\infty}(x)\right)\right)= & \psi\left(\varepsilon_{\alpha}(x)^{*} \varepsilon_{\alpha}(x)\right)-\psi\left(\varepsilon_{\alpha}(x)^{*} \varepsilon_{\infty}(x)\right) \\
& -\psi\left(\varepsilon_{\infty}(x)^{*} \varepsilon_{\alpha}(x)\right)+\psi\left(\varepsilon_{\infty}(x)^{*} \varepsilon_{\infty}(x)\right) .
\end{aligned}
$$

Here the second and third terms of the right-hand side tend to $\psi\left(\varepsilon_{\infty}(x)^{*} \varepsilon_{\infty}(x)\right)$ as $\alpha \uparrow$. Moreover the first term is equal to $\varepsilon_{\alpha}^{*}(\psi)\left(\varepsilon_{\alpha}(x)^{*} x\right)$. Here $\varepsilon_{\alpha}^{*}(\psi)$ tends to $\varepsilon_{\infty}^{*}(\psi)$ as $\alpha \uparrow$ in the norm and $\left\{\varepsilon_{\alpha}(x)\right\}$ is uniformly bounded and converges to $\varepsilon_{\infty}(x)$ in $\sigma$-weak topology. So it follows that $\varepsilon_{\alpha}^{*}(\psi)\left(\varepsilon_{\alpha}(x)^{*} x\right)$ tends to $\varepsilon_{\infty}^{*}(\psi)\left(\varepsilon_{\infty}(x)^{*} x\right)=$ $\psi\left(\varepsilon_{\infty}(x)^{*} \varepsilon_{\infty}(x)\right)$ as $\alpha \uparrow$. Thus $\psi\left(\left(\varepsilon_{\alpha}(x)-\varepsilon_{\infty}(x)\right)^{*}\left(\varepsilon_{\alpha}(x)-\varepsilon_{\infty}(x)\right)\right)$ tends to 0 as $\alpha \uparrow$. $\psi\left(\left(\varepsilon_{\alpha}(x)-\varepsilon_{\infty}(x)\right)\left(\varepsilon_{\alpha}(x)-\varepsilon_{\infty}(x)\right)^{*}\right)$ tends to 0 as $\alpha \uparrow$ in the same way.

In the above theorem the condition that $\varphi$ is semifinite on $\bigcap_{\alpha} N_{\alpha}$ is necessary. Let $M=l^{\infty}$ and for $\left\{x_{i}\right\} \in M_{+}$define $\varphi\left(\left\{x_{i}\right\}\right)=\sum_{i=1}^{\infty} x_{i}$. Then $M$ is a von Neumann algebra and $\varphi$ is a faithful normal semifinite weight on $M_{+}$. Now let $N_{n}$ be the set of all elements $\left\{x_{i}\right\} \in M$ such that $x_{1}=x_{2}=\cdots=x_{n}$. Then $\left\{N_{n}\right\}$ is a decreasing sequence of von Neumann subalgebras of $M$. We can easily see that the conditional 
expectation $\varepsilon_{n}$ onto $N_{n}$ with respect to $\varphi$ exists and for any $\left\{x_{i}\right\} \in M, \varepsilon_{n}\left(\left\{x_{i}\right\}\right)$ is the sequence $\left\{y_{i}\right\}$ such that $y_{i}=(1 / n) \cdot \sum_{k=1}^{n} x_{k}$ for $i=1, \ldots, n$ and $y_{i}=x_{i}$ for $i=n+$ $1, \ldots$ Then $\cap_{n} N_{n}=\{\lambda \cdot I: \lambda \in \mathbf{C}\}$, on which $\varphi$ is not semifinite. Moreover $\left\{\varepsilon_{n}\left(\left\{x_{i}\right\}\right)\right\}$ does not always converge in the $\sigma$-weak topology, because $\left\{(1 / n) \cdot \sum_{k=1}^{n} x_{k}\right\}$ does not always converge.

In the increasing case (resp. the decreasing case) a sequence $\left\{x_{\alpha}\right\}$ is called a martingale if $x_{\alpha} \in N_{\alpha}$ for every $\alpha$ and $\varepsilon_{\alpha}\left(x_{\beta}\right)=x_{\alpha}$ whenever $\alpha \preccurlyeq \beta$ (resp. $\alpha \geqslant \beta$ ). The sequence $\left\{\varepsilon_{\alpha}(x)\right\}$ in Theorem 2 (resp. Theorem 3 ) is an example of martingales and such a martingale is called simple. In the decreasing case any martingale $\left\{x_{\alpha}\right\}$ is essentially simple by considering $\left\{x_{\alpha}\right\}_{\alpha} \geqslant \alpha_{0}$ for any fixed $\alpha_{0}$. In the increasing case, examining the proof of Theorem 2 , we can easily see that a martingale is simple if and only if it is uniformly bounded. Similarly, $L^{1}$-martingales in $M_{*}$ can be considered and it is seen that an increasing $L^{1}$-martingale is simple if it is relatively weakly compact in $M_{*}$.

Recently Accardi and Cecchini [1] generalized the notion of conditional expectations on von Neumann algebras. In our forthcoming paper [8] we investigate strong convergence of martingales in terms of the generalized conditional expectation.

The author would like to express his gratitude to Professor $\mathrm{H}$. Umegaki for his valuable advice and constant encouragement. He also thanks Dr. F. Hiai for useful discussions and suggestions in preparing this manuscript.

\section{REFERENCES}

1. L. Accardi and C. Cecchini, Conditional expectations in von Neumann algebras and a theorem of Takesaki, J. Funct. Anal. 45 (1982), 245-273.

2. A. Connes, On hyperfinite factors of type $\mathrm{III}_{0}$ and Krieger's factors, J. Funct. Anal. 18 (1975). $318-327$.

3. N. Dang-Ngoc, Pointwise convergence of martingales in von Neumann algehras, Isracl J. Math. 34 (1979), 273-280.

4. S. Goldstein, Convergence of martingales in von Neumann algebras, Bull. Acad. Polon. Sci. Sćr. Sci. Math. 27 (1979), 853-859.

5. E. C. Lance, Martingale convergence in von Neumann algebras, Math. Proc. Cambridge Philos. Soc. 84 (1978), 47-56.

6. M. Takesaki, Conditional expectations in von Neumann algebras, J. Funct. Anal. 9 (1972), 306-321.

7. H. Umegaki, Conditional expectation in an operator algebra, Tôhoku Math. J. 6 (1954), 177-181: II, ibid. 8 (1956), 86-100; III, Kōdai Math. Sem. Rep. 8 (1956), 145-151; IV (entropy and information), ibid. 14 (1959), 59-84.

8. F. Hiai and M. Tsukada, Strong convergence of generalized conditional expectations in von Neumann algebras (in preparation).

Department of Information Sciences, Science University of Tokyo, Noda City, Chiba 278, JAPAN 